\title{
Modal Computation of Arbitrary Waveguides Composed of Linear, Circular and Elliptical Arcs
}

\author{
Santiago Cogollos*, Stephan Marini*, Pablo Soto*, \\ Hector Esteban*, Jose V. Morro*, Vicente E. Boria* and Benito Gimeno ${ }^{\dagger}$ \\ ${ }^{*}$ Grupo de Aplicaciones de las Microondas \\ Departamento de Comunicaciones, Universidad Politécnica de Valencia, \\ Camino de Vera s/n, E-46022 Valencia, SPAIN \\ Email: vboria@dcom.upv.es \\ ${ }^{\dagger}$ Dpto. Física Aplicada - I.C.M.U.V. Universidad de Valencia, \\ Dr. Moliner 50, E-46100 Burjassot, Valencia, SPAIN \\ Email: benito.gimeno@uv.es
}

\begin{abstract}
In this paper, the very accurate and efficient modal analysis of arbitrarily shaped wavguides, whose cross-section is defined by a combination of straight, circular and/or elliptical arcs, is solved. An extension of the well-known Boundary Integral-Resonant Mode Expansion (BI-RME) method is proposed. Successful comparisons with available numerical and experimental data fully demonstrates the validity of the improved BI-RME method proposed.
\end{abstract}

\section{INTRODUCTION}

Arbitrary waveguides with a cross-section composed of linear, circular and/or elliptical arcs (see fig. 1) are usually present in many passive waveguide devices [1]. For instance, ridge rectangular or circular waveguides, as well as cross-shaped irises, are frequently used in dual mode empty or dielectric loaded resonator filters. In the same way, the presence of rounded corners in rectangular waveguide passive components, mainly due to the manufacturing techniques presently employed, is already being under investigation. Another example of great practical use is the elliptical waveguide, which has found increasing application in the design of many microwave structures, such as dual and triple mode filters, circular waveguide polarizers, twists and mode launchers, radiators, resonators, and corrugated horns.

Nowadays, most of the CAD tools used in the design of such components require to know, in a very accurate way, the full-wave modal spectrum of the arbitrarily shaped waveguides considered in this paper. Furthermore, the coupling integrals between these modes and those of the standard rectangular waveguide that fully encloses the arbitrary cross-section (see $\Omega$ in fig. 1) must also be known. Among the many methods published in the literature to solve this problem, the BI-RME method originally proposed in [2], [3] has become one of the most popular ones, mainly due to its high speed and accuracy.

The BI-RME methods is based on the solution of an Integral Equation by the Method of Moments, whose practical implementation (see details in [2]) requires to subdivide the boundary contour of the arbitrary waveguide ( $\sigma$ in fig. 1). Even though many efforts have been devoted to improve the BI-RME technique in the last years (see [1], [4]), all

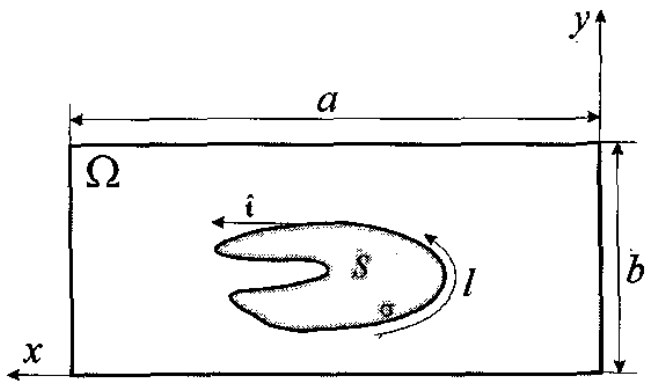

Fig. 1. Arbitrary waveguide with cross section $S$ embedded in a standard rectangular waveguide $\Omega$. Vector $\hat{\mathrm{t}}$ is tangent to $\sigma$ and $l$ is a suitable abscisa taken on the line.

the practical implementations of such technique subdivide the arbitrary contour using only straight segments. In many practical applications, e.g. when circular and/or elliptical arcs are present, the aforementioned subdivision approach based only on straight segments can lead to some errors in the results provided by the BI-RME method. Furthermore, as indicated in [5], such errors are even higher for the lower order modes of the arbitrary waveguides, thus degrading the accuracy of the modal analysis tools.

In this paper, an extension of the BI-RME method, based on a subdivision of the arbitrary contours in rectangular, circular and/or elliptical arcs, is proposed. Several practical results confirming the improved accuracy related to this new technique are offered.

\section{IMPROVING THE TM PROBLEM}

In order to compute the TM modes of an arbitrarily shaped waveguide using the original BI-RME method described in [2], the following matrix elements must be computed

$$
L_{i j}^{\prime}=\int_{\sigma} \int_{\sigma} u_{i}(l) g\left(\mathbf{s}, \mathbf{s}^{\prime}\right) u_{j}\left(l^{\prime}\right) d l d l^{\prime}
$$

where the functions $u_{i}$ and $u_{j}$ are piecewise parabolic basis and weighting functions related to the MoM. The domain of 


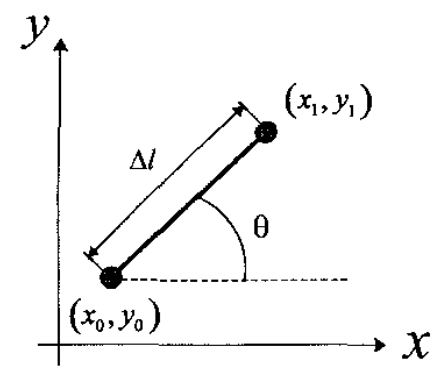

Fig. 2. Arbitrarily oriented straight segment with length $\Delta l$.

these functions are the elements $i$ and $j$ of $\sigma$ that can be straight segments, circular arcs and/or elliptical arcs.

In (1), the double integral is computed numerically with a Gauss quadrature rule, but this is not possible for the diagonal elements of the $\mathbf{L}^{\prime}$ matrix (i.e. when $i=j$ ) because of the singularity of $g$ (the scalar Green function of a cylindrical waveguide). A rapidly convergent expression for $g$ is

$$
g=\frac{1}{4 \pi} \sum_{m=-\infty}^{\infty} \ln \frac{T_{m}^{10} T_{m}^{01}}{T_{m}^{00} \frac{T_{m}^{11}}{T_{m}}}
$$

where

$$
\begin{aligned}
T_{m}^{p q} & =1-2 e^{\tau_{m p}} \cos \frac{\pi}{b}\left(y-(-1)^{q} y^{\prime}\right)+e^{2 \tau_{m p p}} \\
\tau_{m p} & =-\left|x-(-1)^{p} x^{\prime}+2 a m\right| \frac{\pi}{b}
\end{aligned}
$$

The singularity is introduced by the $\ln T_{0}^{00}$ term when the field point $(x, y)$ approaches the source point $\left(x^{\prime}, y^{\prime}\right)$. This term tends to infinity like $\ln R$. In order to treat this singularity, $g$ must be expanded as a Taylor series, and taking into account the following limit

$$
\lim _{\substack{x \rightarrow x^{\prime} \\ y \rightarrow y^{\prime}}} \frac{T_{0}^{00}}{\left(x-x^{\prime}\right)^{2}+\left(y-y^{\prime}\right)^{2}}=\left(\frac{\pi}{b}\right)^{2}
$$

then, the green function can be split in the following way

$$
g=g_{1}-\frac{1}{2 \pi} \ln R
$$

where

$$
\begin{aligned}
& g_{1}=g_{0}-\frac{1}{4 \pi} \ln \frac{T_{0}^{00}}{R^{2}} \\
& g_{0}=\frac{1}{4 \pi} \sum_{\substack{m=-\infty \\
(m \neq 0)}}^{\infty} \ln \frac{T_{m}^{10} T_{m}^{01}}{T_{m}^{00} T_{m}^{11}}+\frac{1}{4 \pi} \ln \frac{T_{0}^{10} T_{0}^{01}}{T_{0}^{11}}
\end{aligned}
$$

and the functions $g_{0}$ and $g_{1}$ are regular everywhere.

Then, the singular part of the diagonal elements of $\mathbf{L}^{\prime}$ matrix is analytically performed. Moreover, this analytic integration is always done in the same limits (in the parameter interval defined by $t \in[-0.5,0.5])$ to fully exploit the code.

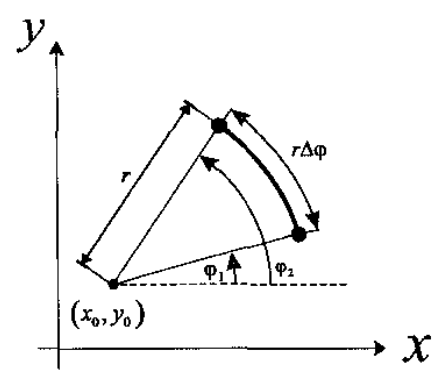

Fig. 3. Arbitrarily oriented circular arc with radius $r$ and length $r \Delta \varphi$.

\section{A. Straight segments}

For straight segments, the integration of the singular term $\ln R$ is straightforward. An arbitrary oriented straight segment can be defined as follows (see fig. 2)

$$
\begin{aligned}
& x=x_{0}+(t+0.5) \Delta l \cos \theta \\
& y=y_{0}+(t+0.5) \Delta l \sin \theta
\end{aligned}
$$

where $t \in[-0.5,0.5]$ and the expression of $\ln R$ is very simple

$$
\ln R^{2}=\ln \left[\left(x-x^{\prime}\right)^{2}+\left(y-y^{\prime}\right)^{2}\right]=\ln \left[\Delta l^{2}\left(t-t^{\prime}\right)^{2}\right]
$$

and the singular integral can be solved immediately because the integration of parabolic functions $u_{i}$ multiplied by $\ln \Delta t^{2}\left(t-t^{\prime}\right)^{2}$ is analytically known.

\section{B. Circular arcs}

Following the same procedure, a circular arc (see fig. 3) can be defined as follows

$$
\begin{aligned}
& x=x_{0}+r \cos \varphi(t) \\
& y=y_{0}+r \sin \varphi(t)
\end{aligned}
$$

where

$$
\begin{aligned}
\varphi(t) & =\varphi_{1}+\Delta \varphi(t+0.5) \\
\Delta \varphi & =\varphi_{2}-\varphi_{1}
\end{aligned}
$$

where $t \in[-0.5,0.5]$ and $r$ is the constant radius of the circular arc. Here, taking into account the Taylor series of $R^{2}, \ln R^{2}$ can be decomposed in two parts

$$
\ln R^{2}=\ln \frac{R^{2}}{\Delta \varphi^{2}\left(t-t^{\prime}\right)^{2}}+\ln \left[\Delta \varphi^{2}\left(t-t^{\prime}\right)^{2}\right]
$$

because the first term of the r.h.s of (14) is regular

$$
\lim _{\substack{x \rightarrow x^{\prime}, y \rightarrow y^{\prime}}} \ln \frac{R^{2}}{\Delta \varphi^{2}\left(t-t^{\prime}\right)^{2}}=\ln r^{2}
$$

With this last result, the regular term in (14) can be added to the regular part of the Green function to be integrated numerically, and the singular term of (14) can be integrated analytically because it is similar to (10). 


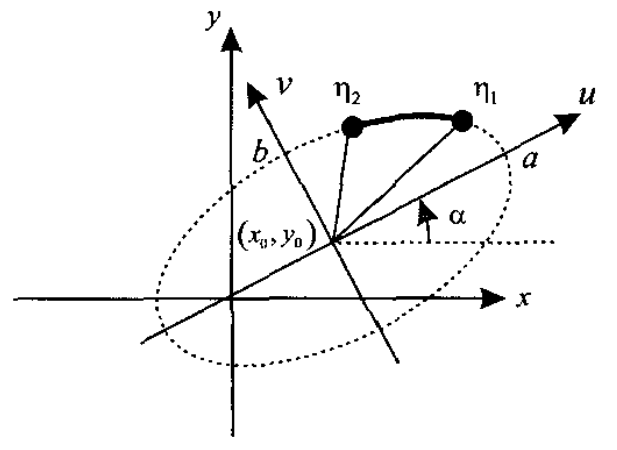

Fig. 4. Arbitrarily oriented elliptic arc.

\section{Elliptical arcs}

Again, following the same procedure of the previous sections, an elliptic arc is defined. In this case, a local coordinate system $(u, v)$ has been chosen (see fig. 4), thus obtaining

$$
\begin{aligned}
& u=a \cos \eta(t) \\
& v=b \sin \eta(t)
\end{aligned}
$$

where

$$
\begin{aligned}
\eta(t) & =\eta_{1}+\Delta \eta(t+0.5) \\
\Delta \eta & =\eta_{2}-\eta_{1}
\end{aligned}
$$

with $t \in[-0.5,0.5]$. Using the cartesian coordinate system $(x, y)$ we obtain

$$
\left(\begin{array}{l}
x \\
y
\end{array}\right)=\left(\begin{array}{l}
x_{0} \\
y_{0}
\end{array}\right)+\left(\begin{array}{cc}
\cos \alpha & -\sin \alpha \\
\sin \alpha & \cos \alpha
\end{array}\right) \cdot\left(\begin{array}{l}
u \\
v
\end{array}\right)
$$

and finally

$$
R^{2}=a^{2}\left[\cos \eta(t)-\cos \eta\left(t^{\prime}\right)\right]^{2}+b^{2}\left[\sin \eta(t)-\sin \eta\left(t^{\prime}\right)\right]^{2}
$$

Now, taking into account the Taylor series of $R^{2}, \ln R^{2}$ can be decomposed in two parts

$$
\ln R^{2}=\ln \frac{R^{2}}{\Delta \eta^{2}\left(t-t^{\prime}\right)^{2}}+\ln \left[\Delta \eta^{2}\left(t-t^{\prime}\right)^{2}\right]
$$

because the first term of the r.h.s part of (20) is regular

$$
\lim _{\substack{x \rightarrow x^{\prime} \\ y \rightarrow y^{\prime}}} \ln \frac{R^{2}}{\Delta \eta^{2}\left(t-t^{\prime}\right)^{2}}=\ln \left[a^{2} \sin ^{2} \eta(t)+b^{2} \cos ^{2} \eta(t)\right]
$$

In this case, the singular term of (20) needs a further treatment in order to obtain an analytical solution.

\section{IMPROVING THE TE PROBLEM}

For the TE problem, the singularities appear in the static part of the bidimensional Green's dyadic $\overline{\mathbf{G}}_{s t}$, whose four components are detailed in [2], and are of the same kind solved before. Therefore, the same procedure described in the previous section can be followed.
In the TE case, an additional problem appears when the dyadic $\overline{\mathbf{G}}_{s t}$ is expanded into a Taylor series, where the following two terms are obtained

$$
\begin{aligned}
\Phi & =\frac{\left(x-x^{\prime}\right)^{2}}{\left(x-x^{\prime}\right)^{2}+\left(y-y^{\prime}\right)^{2}} \\
\Psi & =\frac{\left(x-x^{\prime}\right)\left(y-y^{\prime}\right)}{\left(x-x^{\prime}\right)^{2}+\left(y-y^{\prime}\right)^{2}}
\end{aligned}
$$

which are not singular but discontinuous. This means that the limit when the source point approaches the field point depends on the approximation path. If we have three kinds of segments, these limits must be properly calculated for each case.

\section{A. Straight segments}

If we use (9), the limits of (22) and (23) are easily calculated

$$
\begin{aligned}
& \lim _{\substack{x \rightarrow x^{\prime} \\
y \rightarrow y^{\prime}}} \Phi=\cos ^{2} \theta \\
& \lim _{\substack{x \rightarrow x^{\prime} \\
y \rightarrow y^{\prime}}} \Psi=\frac{1}{2} \sin (2 \theta)
\end{aligned}
$$

\section{B. Circular arcs}

In this case, using (11), the limits of $\Phi$ and $\Psi$ are

$$
\begin{aligned}
& \lim _{\substack{x \rightarrow x^{\prime} \\
y \rightarrow y^{\prime}}} \Phi=\sin ^{2}[\varphi(t)] \\
& \lim _{\substack{x \rightarrow x^{\prime} \\
y \rightarrow y^{\prime}}} \Psi=-\frac{1}{2} \sin [2 \varphi(t)]
\end{aligned}
$$

\section{Elliptical arcs}

In this case, taking (16), the limits of $\Phi$ and $\Psi$ are

$$
\begin{aligned}
\lim _{\substack{x \rightarrow x^{\prime} \\
y \rightarrow y^{\prime}}} \Phi & =\frac{[-a \cos \alpha \sin \eta(t)-b \sin \alpha \cos \eta(t)]^{2}}{a^{2} \sin ^{2} \eta(t)+b^{2} \cos ^{2} \eta(t)} \\
\lim _{\substack{x \rightarrow x^{\prime} \\
y \rightarrow y^{\prime}}} \Psi & =\frac{1}{2} \frac{\chi(t) \sin 2 \alpha-a b \sin 2 \eta \cos 2 \alpha}{a^{2} \sin ^{2} \eta(t)+b^{2} \cos ^{2} \eta(t)}
\end{aligned}
$$

where

$$
\chi(t)=a^{2} \sin ^{2} \eta(t)-b^{2} \cos ^{2} \eta(t)
$$

\section{RESULTS}

This section presents some results that fully validate the extension of the BI-RME method just described. Successful comparisons with other available numerical and experimental data are included.

\section{A. The circular waveguide}

In this example, we compute the cutoff frequencies of the TM modes of a circular waveguide, which is obtained from a standard square contour ( $\Omega$ in fig. 1 ) perturbed by a tubular sheet ( $\sigma$ in fig. 1). Table I compares the relative errors of such cutoff frequencies for the classical BI-RME approach (using only straight segments) and the new extension proposed (considering circular arcs). This benchmark test fully demonstrates the accuracy improvement introduced by the BIRME extension proposed. 
TABLE

RELATJVE ERROR IN THE CUTOFF FREQUENCIES OF THE BI-RME METHOD USING STRAIGHT SEGMENTS AND CIRCULAR ARCS.

\begin{tabular}{|c||c|c|}
\hline $\begin{array}{c}\text { Mode } \\
\text { (TM) }\end{array}$ & $\begin{array}{c}\text { Error BI-RME } \\
\text { (straight segments) }\end{array}$ & $\begin{array}{c}\text { Error BI-RME } \\
\text { (circular segments) }\end{array}$ \\
\hline $\mathrm{TM}_{01}$ & $0.160 \%$ & $0.002 \%$ \\
$\mathrm{TM}_{11}$ & $0.206 \%$ & $0.009 \%$ \\
$\mathrm{TM}_{12}$ & $0.207 \%$ & $0.004 \%$ \\
$\mathrm{TM}_{02}$ & $0.320 \%$ & $0.011 \%$ \\
$\mathrm{TM}_{31}$ & $0.439 \%$ & $0.013 \%$ \\
$\mathrm{TM}_{12}$ & $0.585 \%$ & $0.015 \%$ \\
\hline
\end{tabular}

TABLE II

CUTOFF WAVENUMBERS USING BI-RME WITH ELLIPTICAL ARCS VERSUS THE METHOD OUTLINED IN [6]

\begin{tabular}{|c||c|c|c|}
\hline $\begin{array}{c}\text { Mode } \\
\text { (TE/TM) }\end{array}$ & $\begin{array}{c}k_{c} \\
\text { ref. [6] }\end{array}$ & $\begin{array}{c}k_{c} \\
\text { (elliptical segments) }\end{array}$ & $\begin{array}{c}\text { Relative error } \\
(\%)\end{array}$ \\
\hline TE & 0.370200 & 0.370256 & 0.015 \\
TE & 0.422472 & 0.422540 & 0.016 \\
TM & 0.519355 & 0.519450 & 0.018 \\
TE & 0.644532 & 0.644830 & 0.04 \\
TE & 0.658631 & 0.659039 & 0.06 \\
TM & 0.797281 & 0.797679 & 0.04 \\
\hline
\end{tabular}

\section{B. The elliptical waveguide}

Next, an elliptical waveguide defined within a rectangular WR-90 waveguide has been considered. Here, $\sigma$ is an ellipse of eccentricity equal to 0.5 and semi-major axis equal to $5 \mathrm{~mm}$. Table II successfully compares the cutoff wavenumbers provided by this method with results from [6].

\section{Filter analysis}

As a final example of practical use, a four-pole inductively coupled rectangular waveguide filter in with rounded comers has been designed, manufactured and measured. The rounded corners that usually appear in the manufacturing process have been accurately considered using the extended BI-RME method proposed in this paper. As can be seen in fig. 5, this filter is composed of several pieces that can be manufactured using low cost milling. Figure 6 shows a very good agreement between simulated and measured'results.

\section{CONCLUSION}

In this paper, an extension of the BI-RME method that can handle straight, circular and elliptical segments in the discretization of arbitrary cross section waveguides is presented. The validation of this extended technique has been carried out with numerical and experimental results. Finally, this method has been used in the design process of a rectangular waveguide filter with rounded corners. The simulated results have been successfully compared with measurements of a low cost manufactured prototype.

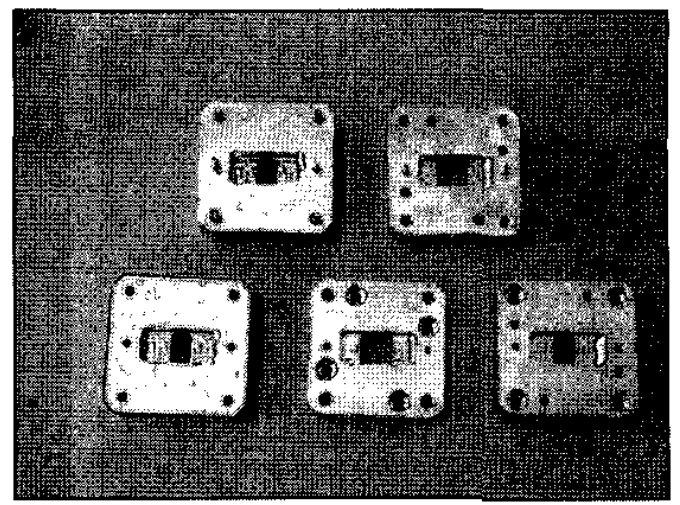

Fig. 5. Pieces of the four pole inductive filter with rounded corners.

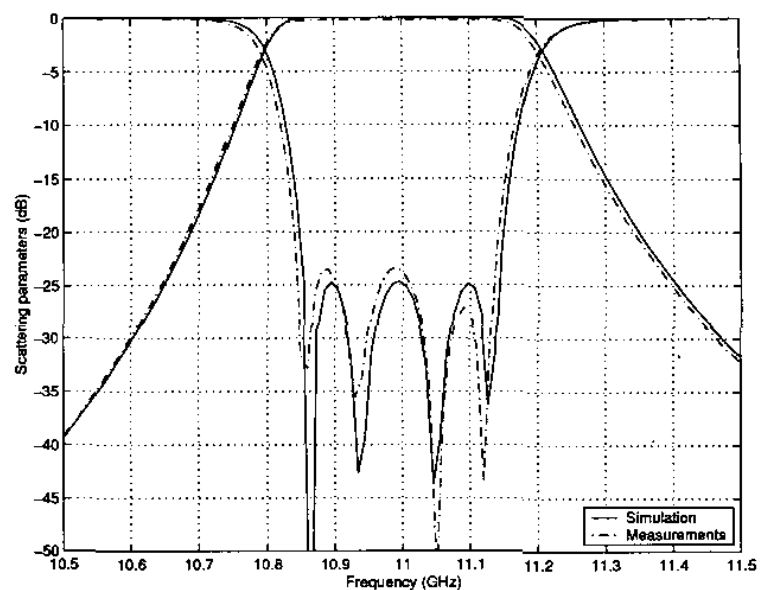

Fig. 6. Simulated and measured S-parameters of the four pole inductive filter with rounded corners.

\section{ACKNOWLEDGMENT}

This work has been supported by European Commission under the Research and Training Networks Programme, Contract No. HPRN-CT-2000-00043.

\section{REFERENCES}

[1] G. Conciauro, M. Guglielmi, and M. Sorrentino, Advanced Modal Analysis - CAD Techniques for Waveguide Components and Filters. Chichester: John Wiley \& Sons, Ltd., 2000.

[2] G. Conciauro, M. Bressan, and C. Zuffada, "Waveguide modes via an integral equation leading to a linear matrix eigenvalue problem," IEEE Trans. Microwave Theory Tech., vol. 32, no. 11, pp. 1495-1504, November 1984

[3] P. Arcioni, "Fast evaluation of modal coupling coefficients of waveguide step discontinuities," IEEE Microwave Wireless Compon. Lett., vol. 6, no. 6 , pp. 232-234, June 1996

[4] M. Bozzi, G. Conciauro, and L. Perregrini, "On the evaluation of modal coupling coefficients by contour integrals," IEEE Trans. Microwave Theory Tech., vol. 50, no. 7, pp. 1853-1855, July 2002.

[5] R. Courant and D. Hilbert, Methods of Mathematical Physics, ser. Wiley Classics Library. New York: John Wiley \& Sons Wiley-Interscience, 1989, vol. I.

[6] B. Gimeno and M. Guglielmi, "Full wave network representation for rectangular, circular, and elliptical to elliptical waveguide juctions," IEEE Trans. Microwave Theory Tech., vol. 45, no. 3, pp. 376-384, March 1997. 